\title{
O “LUGAR” DAS RELAÇÕES PÚBLICAS NA INDÚSTRIA CRIATIVA
}

\author{
The "place" of Public Relations in the Creative Industry \\ El "lugar" de las Relaciones Públicas en la Industria Creativa
}

Tiago Costa Martins

Professor da Universidade Federal do Pampa

tiagominuano@gmail.com

\begin{abstract}
Resumo
A comunicação encontra, na indústria criativa, um campo profícuo para discussões científicas. Às relações públicas a problemática recai em percebê-la enquanto ciência ou arte e em posicioná-la na indústria criativa. A presente pesquisa, exploratória, bibliográfica e documental objetiva perceber tal posicionamento, discutindo a metodologia do Department for Culture, Media and Sport do Reino Unido. Conclui-se o artigo argumentando que as relações públicas, a partir das práticas, da ocupação profissional e da atividade econômica, são creative service providers da indústria criativa.
\end{abstract}

Palavras-chaves: Indústria Criativa; Relações Públicas; Creative Service Providers.

\begin{abstract}
Communication finds in the creative industry a profitable field for scientific discussions. Regarding, public relations the problem lies in perceiving it as a science or art and in positioning it in the creative industry. The present exploratory, bibliographical and documentary research, aims to perceive such positioning, discussing the methodology of the Department for Culture, Media and Sport of the United Kingdom. The article concludes by arguing that public relations, based on practices, professional occupation and economic activity, are creative service providers of the creative industry.
\end{abstract}

Keywords: Creative Industry; Public relations; Creative Service Providers.

\section{Resumen}

La comunicación encuentra en la industria creativa un campo proficuo para discusiones científicas. A las relaciones públicas la problemática recae en percibirla como ciencia o arte y en posicionarla en la industria creativa. La presente investigación exploratoria, bibliográfica y documental, objetiva distinguir tal posicionamiento, discutiendo la metodología del Department for Culture, Media and Sport de Reino Unido. Se concluye el artículo argumentando que las relaciones públicas, a partir de las prácticas, de la ocupación profesional y de la actividad económica, son creative service providers de la industria creativa.

Palabras-claves: Industria Creativa; Relaciones Públicas; Creative Service Providers. 


\section{INTRODUÇÃO}

As análises em torno da indústria criativa encontram, na comunicação, um campo profícuo para discussões científicas que levam em conta as conexões e as possibilidades de articulação do jornalismo, audiovisual, publicidade e relações públicas nesse setor. Isso se faz possível especialmente, após o adensamento nos estudos da indústria criativa, a ampliação do financiamento enquanto políticas públicas - ênfase na União Europeia, bem como na divulgação crescente dos impactos, positivos, do setor na economia global e nacional ${ }^{1}$.

Dentro do reconhecimento da indústria criativa como a configuração de setores produtivos que têm, como insumo básico, uma dimensão imaterial, simbólica e criativa, é possível perceber, de antemão, as profissões da comunicação em dois aspectos relacionados: comunicação para $a$ indústria criativa e comunicação como indústria criativa. $\mathrm{O}$ primeiro se estabelece a partir de um domínio técnico de um saber-fazer específico, os profissionais da comunicação podem organizar/apoiar as atividades criativas e culturais; o segundo, quando se reconhece que os produtos gerados pelas profissões da comunicação são produtos criativos oriundos de uma produção intelectual (PPGCIC, 2018).

No entanto, quando o tema dessa articulação recaía sobre as relações públicas, paira, acredita-se, uma indefinição no debate. O trabalho do profissional de relações públicas é para $a$ ou como indústria criativa? O produto gerado é indústria criativa? Ou o relações públicas é um agente mediador dos processos criativos? Em grande medida, a problemática é posicionar as relações públicas no setor, perguntando: qual o lugar das relações públicas na indústria criativa?

As discussões que se seguem são reconhecidas como de cunho exploratório por meio de pesquisa bibliográfica e documental ${ }^{2}$ e objetivam perceber as relações públicas na indústria criativa. Para isso, três momentos são recorridos com essa intenção: primeiro, apresentar as relações públicas num breve debate sobre a atuação profissional; segundo, compreender a

\footnotetext{
${ }^{1} \mathrm{Na}$ Europa, o trabalho desenvolvido por centros de pesquisa, tal como a Organisation for Economic Cooperation and Development (OECD) com o The Cultural and Creative Cities Monitor (https://goo.gl/R1EDbh); ou o programa de investimentos da União Europeia intitulado Creative Europe (https://goo.gl/SSJNUU), são exemplos possíveis.

${ }^{2}$ A pesquisa exploratória foi desenvolvida por ocasião das atividades de pós-doutoramento do investigador no Mestrado em Comunicação e Gestão das Indústrias Criativas, Faculdade de Letras, Universidade do Porto, Portugal.
} 
indústria criativa como conceito e categorização; terceiro, apresentar alguns pontos que localizam as relações públicas na indústria criativa.

Dessa forma, os tópicos do presente artigo recorrerão brevemente ao conceito de relações públicas, enfatizando o debate entre arte e ciência na configuração da atividade. Após, trará um entendimento para indústria criativa, centrando sua compreensão no conceito e na construção analítica do Department for Culture, Media and Sport (DCMS, 2016) do Reino Unido. Os dois tópicos seguintes procuram associar relações públicas na indústria criativa: um, pelo viés analítico do modelo do DCMS; outro, mais teórico conceitual, com alguns autores do tema. Por fim, o último tópico é conclusivo, no sentido de ordenar os entendimentos em busca de uma resposta adequada para o lugar das relações públicas na indústria criativa.

\section{AS RELAÇÕES PÚBLICAS ENTRE A ARTE E A CIÊNCIA}

Não é objetivo deste estudo apresentar uma significativa discussão sobre a definição de relações públicas. No entanto, sabe-se que, diante de propostas focadas na gestão, no binômio organização-públicos, na imagem etc., o mais salutar a este estudo é entender que as relações públicas "são responsáveis por gerir a relação de uma organização com os diferentes actores sociais (...) através da comunicação planificada" (GONÇALVES, 2013, p.21). Isto, no momento, é suficiente para a pretensão de articular relações públicas e indústria criativa.

Eis, então, que, numa passagem histórica das relações públicas, Edward Bernays (1891-1995) disse que seu trabalho se baseava na ciência, enquanto o de Ivy Lee (1877-1934) na arte (GONÇALVES, 2013). No contexto dessa afirmação, estava presente um Ivy Lee com sua política de franqueza, muito mais um conselheiro do que um técnico-científico. Como ele sentenciou, por ocasião de um acidente de trem da Pennsylvania Railroad, seu trabalho era interpretar a empresa para o público e o público para a ferrovia. "Seus conselhos para a administração se fundamentavam na sua intuição sobre a opinião pública” (GRUNIG; HUNT, 2003, p. 92, tradução nossa) ${ }^{3}$.

De outro lado, havia um Bernays preocupado em conferir às relações públicas um status de ciência social aplicada, com alto teor de instrumentalidade, práticas e procedimentos

\footnotetext{
${ }^{3}$ Tradução livre para: "Sus consejos a la dirección se fundamentaban en su intuición sobre la opinión pública”.
} 
padronizados. A engenharia de consenso de Bernays consistia numa construção profissional de uma engenharia social científica, baseada em estratégias contínuas e sistemáticas de controle e regulação dos públicos. Elucidativamente,

\begin{abstract}
(...), Bernays colocava no centro das suas estratégias persuasivas as mensagens indirectas. Quer isto dizer que selecionava criteriosamente líderes de opinião, especialistas ou figuras públicas para emitirem mensagens favoráveis à empresa ou aos seus produtos em notícias, reportagens ou artigos de opinião. Conseguia vender pianos Mozart, por exemplo, não porque fazia publicidade à marca, mas porque desenvolvia a aceitação pública da ideia de que ter uma sala de música em casa era sinónimo de status social (GONÇALVES, 2013, p. 59).
\end{abstract}

Por essa ordem, há, para Bernays, o entendimento de que as relações públicas são a aplicação prática do conhecimento das ciências sociais. Além disso, a atividade é planejada e executada por profissionais treinados de acordo com princípios científicos, baseados em tais ciências. Para ele, as relações públicas são uma engenharia do consenso (TURNEY, 2009).

No entanto, alguns questionamentos importantes são levantados na atualidade e merecem uma apresentação:

\begin{abstract}
As relações públicas como disciplina desenvolveram práticas e procedimentos padronizados e amplamente aceitos? Essas práticas e procedimentos produzem resultados consistentes cada vez que são usados e o resultado das atividades de relações públicas pode ser previsto com um grau razoável de precisão estatística? (TURNEY, 2009, tradução nossa) ${ }^{4}$.
\end{abstract}

Uma resposta positiva leva diretamente ao pensamento: relações públicas mecanicista, científica e previsível. Mas há margem para se pensar que a dose de cientificismo e de racionalidade, já em Bernays, não encontrou unanimidade na época e, diga-se, na atualidade também. Os profissionais não acreditavam que relações públicas fossem mecanicistas ou que sua funcionalidade fosse baseada na certeza e na previsão. Havia muita incerteza, em todos os esforços de relações públicas, e a capacidade de prever o resultado das atividades foi relativizada por muitos profissionais (TURNEY, 2009).

Reconhece-se que a atividade realiza análises cuidadosas, usa técnicas de pesquisas sofisticadas, dispõe de métricas aprimoradas para aferição; mas, no dizer de Kirk Hazlett (2012), há uma arte na forma como se desenvolvem os programas de relações públicas e

\footnotetext{
${ }^{4}$ Tradução livre para: "Has public relations as a discipline developed standardized and widely-accepted practices and procedures? Do these practices and procedures reliably produce consistent results each time they're used, and can the outcome of public relations activity be predicted with a reasonable degree of statistical accuracy?".
} 
criam-se as mensagens. Pensamento também compartilhado por Turney (2009), que percebe as relações públicas cada vez mais baseadas no conhecimento, mais mensuráveis e mais responsáveis com o tempo, mas que sempre estarão associadas com a imprevisibilidade e a contrariedade da natureza humana.

\begin{abstract}
Porque lida com as pessoas, as interações e relacionamentos que eles têm um com o outro, as relações públicas nunca serão uma ciência exata. Sua prática permanecerá para sempre uma arte, uma estrutura ou uma habilidade. Continuará a ter regras e diretrizes, técnicas verdadeiras e padrões de excelência, mas nunca serão totalmente codificados. Nem a intuição e sentimentos humanos serão totalmente eliminados de sua prática. Mas, quanto mais sofisticados, mais experientes e mais humanos seus praticantes se tornam, mais artístico, elegante e eficaz será a sua prática (TURNEY, 2009 , tradução nossa) $)^{5}$.
\end{abstract}

Essa dupla associação - ciência e arte - em relações públicas é apresentada por Hazlett (2012) nos conceitos fundamentais da atividade pela Public Relations Society of America: comunicação estratégica, com um planejamento cuidadoso e calculado, que se utiliza de pesquisa (ciência); construção de relacionamentos mutuamente benéficos, orquestrando acordos tácitos e expressos (arte). Assim, “construir e manter relacionamentos é peça chave, e ambos os aspectos artístico e científico desempenham partes igualmente importantes" (HAZLETT, 2012, tradução nossa) ${ }^{6}$.

Dessa forma, o pensamento de Bernays percorreu os anos com continuidades e rupturas na sua proposição. Nota-se que é possível reconhecer as relações públicas a partir da combinação cuidadosa entre a ciência e arte. Waldermar Kunsch (2006), em seu estudo sobre o centenário das relações públicas (1906-2006), argumenta que, reconhecidas as contribuições de Ivy Lee para a projeção da atividade profissional e de Edward Bernays para o campo científico, é necessário adequar esses paradigmas, seus conceitos e as suas práticas, para que as relações públicas possam acompanhar as transformações econômicas e tecnológicas da contemporaneidade. Este breve debate permite o avanço para o tema tão atual da indústria

\footnotetext{
${ }^{5}$ Tradução livre para: "Because it deals with people and the interactions and relationships they have with one another, public relations will never be an exact science. Its practice will forever remain an art, a craft, or a skill. It will continue to have rules and guidelines, tried and true techniques, and standards of excellence, but they will never be fully codified. Nor will human intuition and feelings ever be totally eliminated from its practice. But, the more sophisticated, more experienced, and more human its practitioners become, the more artistic, elegant, and effective its practice will be".

${ }_{6}$ Tradução livre para: "Building and maintaining relationships is key, and both the artistic and the scientific aspects play equally important parts".
} 
criativa. A sequência do estudo procurará sugerir o lugar das relações públicas na indústria criativa.

\section{UM ENTENDIMENTO DE INDÚSTRIA CRIATIVA}

O debate sobre indústria criativa pode ser considerado recente. Marcado pela passagem da economia baseada no uso intensivo de capital e trabalho para uma economia de base intelectual fundamentada no indivíduo (BENDASSOLLI et al., 2009), a expressão surge na Austrália, em 1994, e ganha pressupostos políticos e econômicos no Reino Unido, em 1997 (UNCTAD, 2010).

Seu entendimento deve reconhecer quatro componentes principais: criatividade é um elemento central; cultura é tratada como objeto cultural, reconhecendo os sentidos compartilhados e a utilidade gerada; esses sentidos são transformados em propriedade intelectual; há uma convergência entre artes, negócios e tecnologia (BENDASSOLLI, et al., 2009). Articulados, tais componentes levam às características das indústrias criativas:

o primeiro bloco refere-se a uma forma de produção que tem a criatividade como recurso-chave, que valoriza a arte pela arte, que fomenta o uso intensivo de novas tecnologias de informação e de comunicação, fazendo uso extensivo de equipes polivalentes; o segundo bloco abrange os contornos específicos dos produtos gerados, tais como a variedade infinita, a diferenciação vertical e a perenidade; e o terceiro bloco representa uma forma particular de consumo, que possui caráter cultural e apresenta grande instabilidade na demanda (BENDASSOLLI et al., 2009, p. 11).

Trata-se, portanto, de perceber que a indústria criativa diz respeito a um conjunto de setores com especificidades no tipo de insumo, na sua forma de transformação, no produto gerado e no consumo realizado. Sinteticamente, a criatividade é o insumo, e o conteúdo é o produto.

Dessa forma, alguns conceitos para indústria criativa podem ser percebidos, como o de Howkins (2005, p. 119 apud BENDASSOLLI et al., 2009, p. 12), que a entende como "uma indústria onde o trabalho intelectual é preponderante e onde o resultado alcançado é a propriedade intelectual". Já para a Conferência das Nações Unidas para o Comércio e o Desenvolvimento - Unctad, a indústria criativa é percebida como:

- (...) os ciclos de criação, produção e distribuição de produtos e serviços que utilizam criatividade e capital intelectual como insumos primários; 
- constituem um conjunto de atividades baseadas em conhecimento, focadas, entre outros, nas artes, que potencialmente geram receitas de vendas e direitos de propriedade intelectual;

- constituem produtos tangíveis e serviços intelectuais ou artísticos intangíveis com conteúdo criativo, valor econômico e objetivos de mercado;

- posicionam-se no cruzamento entre os setores artísticos, de serviços e industriais;

- constituem um novo setor dinâmico no comércio mundial. (UNCTAD, 2010, p. 08).

O entendimento de indústria criativa para a Unctad está nas interligações entre artes, negócios e tecnologia, incluindo-se atividades culturais tradicionais (dança, música, etc.) e atividades mais ligadas ao mercado (editorial, publicidade, mídias) (VALIATI, et al., 2017). Assim, a classificação do órgão está dividida em quatro grandes grupos: patrimônio, artes, mídia e criações funcionais. O esquema abaixo apresenta esses grupos com seus respectivos subgrupos.

Figura 01 - Classificação da Unctad para as indústrias criativas (2010).

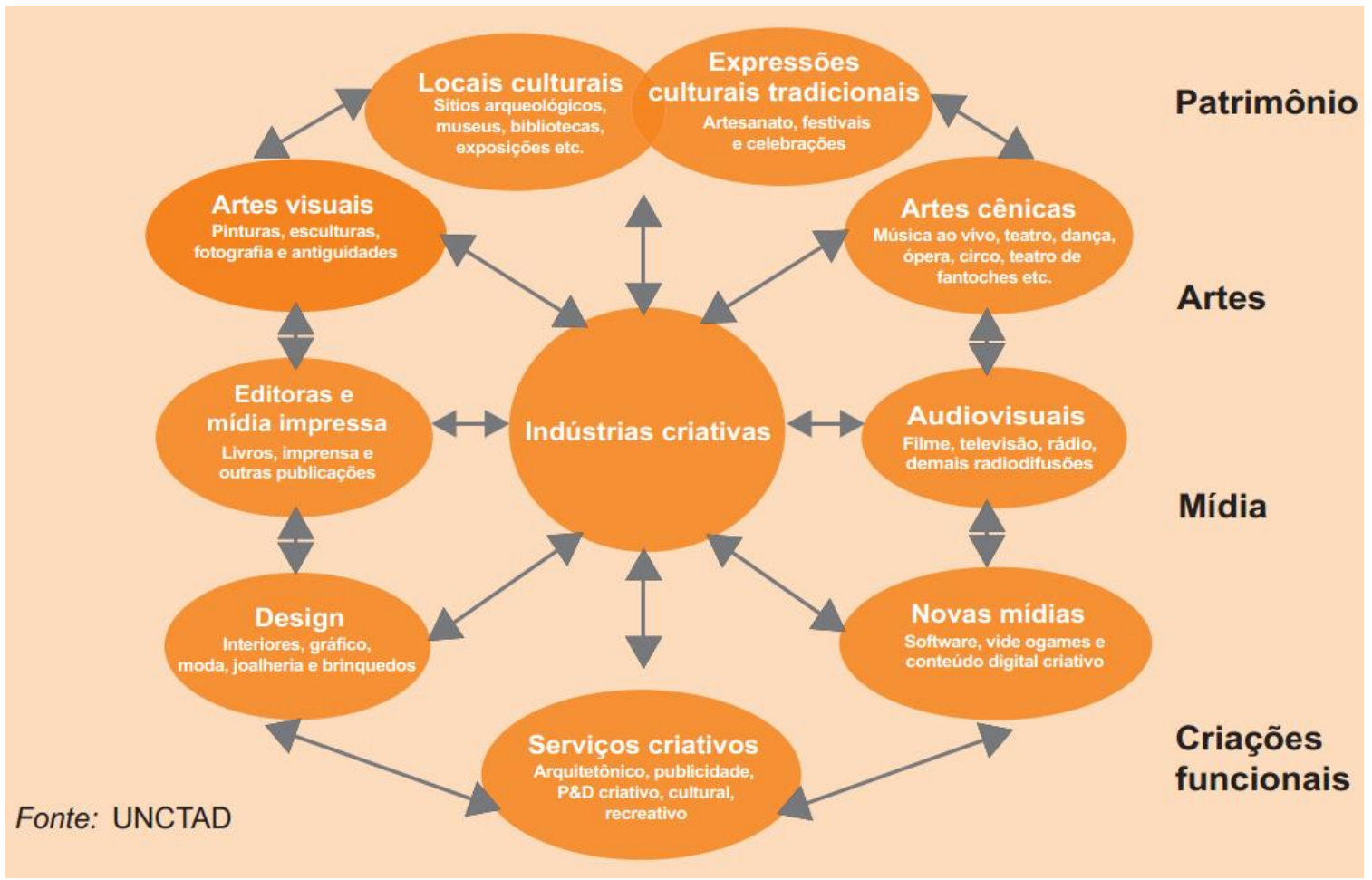

O modelo da Unctad cumpre a função de perceber essas atividades no contexto das trocas econômicas de bens e serviços no âmbito internacional. Em grande medida, enfatiza o produto gerado e o consumo realizado pela dimensão da troca econômica. 
Por seu turno, Throsby (2008, p. 148, tradução nossa), na configuração do modelo dos círculos concêntricos, confere centralidade ao valor cultural dos bens e serviços. "O modelo afirma que é o valor cultural, ou conteúdo cultural, dos bens e serviços produzidos que confere às indústrias culturais sua maior característica distinguida"7. $\mathrm{O}$ modelo constrói uma estrutura hierárquica em que as ideias criativas são nucleares: quanto mais nítido o conteúdo cultural de um bem ou serviço, mais central será a posição da indústria que o produz; ainda: à medida que essas ideias vão se combinando com outros insumos e incorporam outros processos, alteram-se as dimensões de valor: do cultural para o comercial.

\begin{abstract}
Assim os círculos concêntricos são delineados: no centro estão as indústrias cuja proporção de conteúdo cultural para comercial é julgado de acordo com critérios determinados para ser mais alta, com camadas que se estendem para fora do centro à medida que o conteúdo cultural cai em relação ao valor comercial dos produtos ou serviços produzidos (THROSBY, 2008, p. 149, tradução nossa) ${ }^{8}$.
\end{abstract}

Resumidamente, para o teórico, quanto mais centrais as atividades criativas e culturais, mais valor de conteúdo cultural - como literatura, música, dança, dentre outros - elas terão. Quanto mais periféricas as atividades que envolvem insumos e outros processos - como propaganda, design, moda, dentre outros - mais comerciais elas serão. O esquema abaixo sintetiza o pensamento de Throsby (2008).

Figura 02 - Modelo círculos concêntricos Throsby (2008).

\footnotetext{
7 Tradução livre para: "The model asserts that it is the cultural value, or cultural content, of the goods and services produced that gives the cultural industries their most distinguishing characteristic".

${ }^{8}$ Tradução livre para: "Thus are the concentric circles delineated: at the centre are core industries whose proportion of cultural to commercial content is judged according to given criteria to be highest, with layers extending outwards from the centre as the cultural content falls relative to the commercial value of the commodities or services produced".
} 


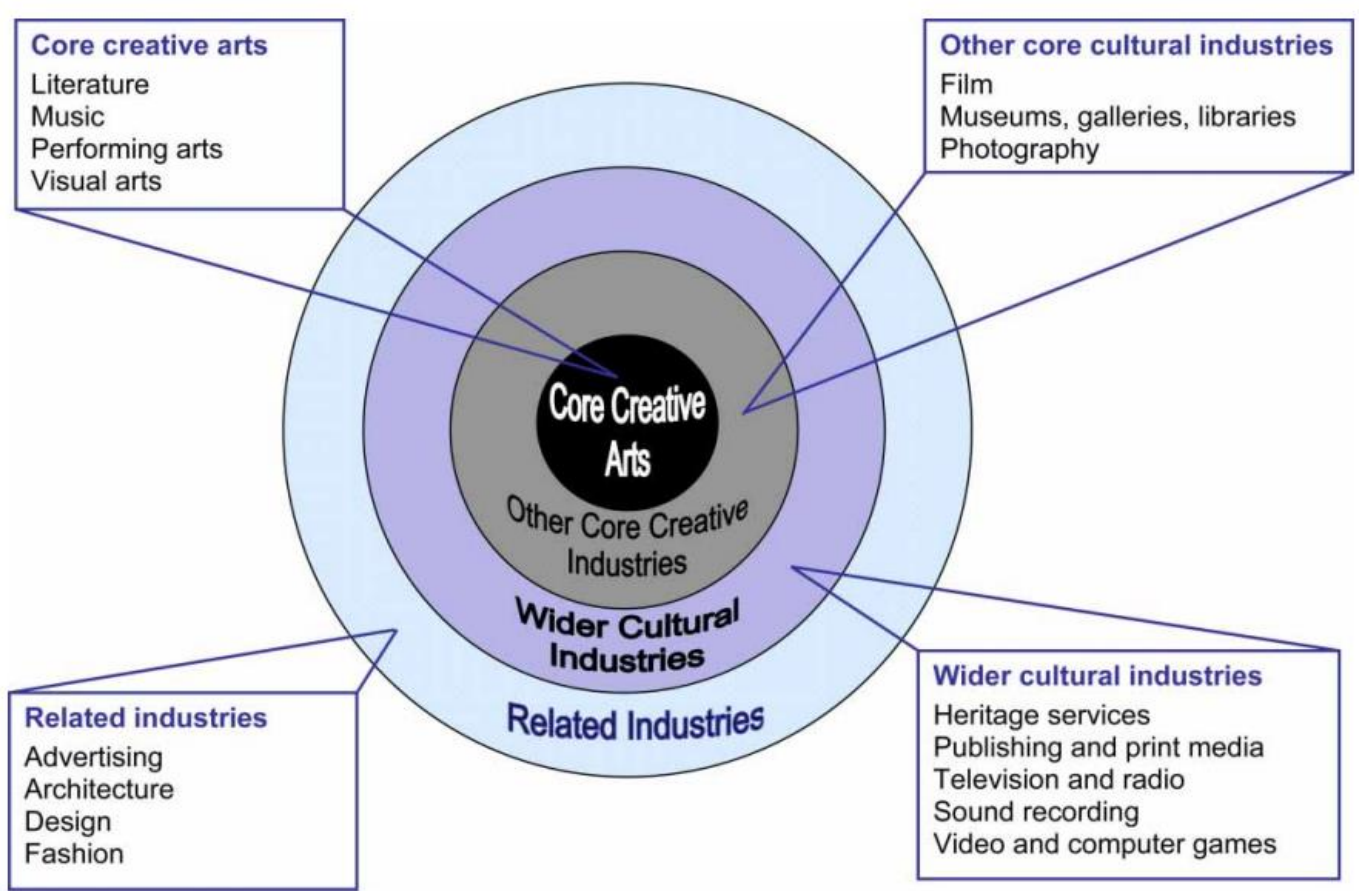

Fonte: Throsby (2008, p. 150).

Ao enfatizar a dimensão do valor, nota-se que essa perspectiva destaca o processo de transformação do insumo (criatividade como insumo "puro" até sua associação com outros insumos e aplicação de diferentes processos) em produto gerado, particularmente pela configuração do seu valor (conteúdo cultural/econômico).

No entanto, o terceiro e último entendimento de indústria criativa que interessa a este estudo é o definido pelo Department for Culture, Media and Sport - DCMS, do Reino Unido. Para o órgão, a indústria criativa são "aquelas indústrias que têm sua origem na criatividade, habilidade e talento individual e que têm potencial para riqueza e criação de emprego através da geração e exploração da propriedade intelectual” (DCMS, 2016, p. 3, tradução nossa) ${ }^{9}$.

A partir desse conceito, o DCMS aponta um caminho relevante à compreensão de indústria criativa, construção aqui considerada importante para o posicionamento da atividade de relações públicas na indústria criativa. Ao contrário dos modelos anteriores, a proposta do Reino Unido entende que o trabalhador criativo é o insumo mais importante na indústria criativa (VALIATI, et al., 2017), pois criatividade, habilidade e talento partem do indivíduo.

Para indicar a indústria criativa, o departamento separa as ocupações criativas e as atividades criativas. O primeiro, ocupações, é oriundo do Standard Occupational

\footnotetext{
${ }^{9}$ Tradução livre para: "those industries which have their origin in individual creativity, skill and talent and which have a potential for wealth and job creation through the generation and exploitation of intellectual property".
} 
Classification - SOC, um meio de classificar a ocupação de uma pessoa de acordo com o trabalho que ela faz e o nível de habilidade requerido. Já as atividades criativas são retiradas da Standard Industrial Classification - SIC, uma forma de classificar as empresas de acordo com o tipo de atividade econômica em que estão envolvidos (DCMS, 2016).

Após selecionar as ocupações criativas, o DCMS estabelece uma relação entre a força de trabalho nas ocupações e as atividades criativas. A isso designou o nome de intensidade criativa.

\begin{abstract}
A metodologia compreende 3 etapas: 1 . Um conjunto de ocupações é identificado como criativo. 2. A intensidade criativa é calculada para todas as indústrias da economia. 3. Todas as indústrias com uma intensidade criativa acima de certo "limite" são classificadas como Indústrias Criativas (DCMS, 2016, p. 36, tradução nossa) ${ }^{10}$.
\end{abstract}

O fato interessante dessa abordagem ocupacional do Reino Unido é a compreensão de que há profissões criativas dentro das atividades criativas, mas também há profissões criativas em outras atividades não consideradas assim. Por exemplo, publicitários trabalham em agências de publicidade, mas também podem trabalhar em empresas automobilísticas. Para tal situação, o DCMS (2016) faz uma distinção entre economia criativa e indústria criativa: a primeira inclui o contributo de todas as profissões criativas que estão fora da indústria criativa, bem como o de todas aquelas empregadas na indústria criativa; já indústria criativa inclui apenas aquelas ocupações que trabalham nas próprias indústrias criativas (como atividades - SIC). Objetivamente, "as indústrias que tiveram mais de 6.000 empregos e uma 'intensidade criativa' de mais de 30 por cento foram consideradas candidatas à inclusão [como indústria criativa]" (DCMS, 2016, p. 19, tradução nossa) ${ }^{11}$.

Assim, a partir das intensidades criativas dos setores econômicos, definiram-se 31 códigos de atividade econômica, separados por nove grupos, também separados pelos mesmos grupos das ocupações (VALIATI; et al., 2017). O quadro abaixo apresenta a categorização do modelo.

Quadro 01 - Indústrias criativas modelo DCMS/UK (2016).

\title{
Grupos
}

\footnotetext{
${ }^{10}$ Tradução livre para: The methodology comprises 3 steps. 1. A set of occupations are identified as creative. 2. Creative intensity is calculated for all industries in the economy. 3. All industries with a creative intensity above a certain "threshold" are classified as Creative Industries.

11 Tradução livre para: Industries which had more than 6,000 jobs and a "creative intensity" of more than 30 per cent were considered as candidates for inclusion.
} 


\begin{tabular}{|l|}
\hline Publicidade e marketing \\
\hline Arquitetura \\
\hline Artesanato \\
\hline Design: produto, gráfico e moda \\
\hline Filme, TV, vídeo, rádio e fotografia \\
\hline TI, software e serviços de informática \\
\hline Editorial \\
\hline Museus, galerias e bibliotecas \\
\hline Música, artes performáticas e artes visuais \\
\hline
\end{tabular}

Fonte: Valiati; et al.(2017) (com adaptações).

Em síntese, os modelos de indústria criativa aqui apresentados destacaram: (i) as interligações entre artes, negócios e tecnologia e as atividades mais ligadas ao mercado; (ii) a dimensão do valor, enfatizando o processo de transformação da criatividade como insumo "puro" até sua associação com outros insumos e aplicação de diferentes processos; (iii) a dimensão ocupacional na qual o trabalhador criativo é o insumo mais importante na indústria criativa, enfatizando a articulação entre ocupação e atividade na configuração da indústria criativa. Para o caso das relações públicas, é particularmente esse último que será utilizado. Assim, o tópico a seguir procurará posicionar a profissão e a atividade de relações públicas na indústria criativa.

\section{RELAÇÕES PÚBLICAS NA INDÚSTRIA CRIATIVA}

É no modelo do DCMS que se encontrarão explicitamente as relações públicas como indústria criativa. A descrição objetiva do DCMS (2001) é apresentada no Mapping Documents para o grupo de indústria criativa "publicidade e marketing". Nota-se, nesse documento, que as atividades nucleares do grupo são apresentadas como pesquisa do consumidor, elaboração de planos de comunicação, campanhas de relações públicas e planejamento de mídia. Atividades associadas à atuação das relações públicas e que se aproximam do fazer criativo, como aqueles setores que têm sua origem na criatividade individual, habilidade e talento, estão, por sua vez, associadas à propriedade intelectual de quem a constitui e a produz.

No entanto, é na apresentação da metodologia do DCMS, baseada na ocupação e na atividade, que as relações públicas são consideradas indústria criativa no grupo da "publicidade e marketing”. O Standard Occupational Classification - SOC indica a existência 
da ocupação relações públicas, e o DCMS reconhece essa ocupação como sendo criativa, conforme quadro abaixo.

Quadro 02 - Grupo de ocupações criativas DCMS (2016).

\begin{tabular}{|l|l|l|}
\hline $\begin{array}{l}\text { Grupo de ocupações } \\
\text { criativas }\end{array}$ & $\begin{array}{l}\text { SOC } \\
\mathbf{( 2 0 1 0 )}\end{array}$ & Descrição \\
\hline Publicidade e Marketing & 1132 & Diretores de marketing e vendas \\
\cline { 2 - 3 } & $\mathbf{1 1 3 4}$ & $\begin{array}{l}\text { Diretores de publicidade e relações } \\
\text { públicas }\end{array}$ \\
\cline { 2 - 3 } & $\mathbf{2 4 7 2}$ & Profissionais de relações públicas \\
\cline { 2 - 3 } & 2473 & $\begin{array}{l}\text { Gerentes de contas publicitárias e diretores } \\
\text { criativos }\end{array}$ \\
\cline { 2 - 3 } & 3543 & Profissionais associados ao marketing \\
\hline
\end{tabular}

Fonte: DCMS (2016, p. 21), com adaptações. [grifos nosso].

Aqui, então, o trabalho de relações públicas é percebido como uma ocupação criativa, desenvolvida por um profissional de acordo com um nível de habilidade específico. O código SOC 2472 é explícito nesses termos ao categorizar o profissional de relações públicas. Além disso, dos cinco códigos de ocupação no grupo, duas trazem diretamente as relações públicas como ocupação.

Por seu turno, a categorização do DCMS pelo viés das atividades também permite reconhecer as relações públicas como uma atividade criativa percebida a partir do Standard Industrial Classification - SIC. O quadro abaixo apresenta as atividades do Reino Unido para o setor, delineando o que se reconhece como indústria criativa no grupo de "publicidade e marketing".

Quadro 03 - Indústria Criativa no grupo “publicidade e marketing”, DCMS (2016).

\begin{tabular}{|l|l|lll|}
\hline Grupo & $\begin{array}{l}\text { SIC } \\
\mathbf{( 2 0 0 7 )}\end{array}$ & Descrição & \\
\hline Publicidade e marketing & 70.21 & $\begin{array}{l}\text { Relações públicas e atividades de } \\
\text { comunicação }\end{array}$ & \\
\cline { 2 - 5 } & 73.11 & Agências de propaganda & \\
\cline { 2 - 4 } & 73.12 & Meios de comunicação (mídia) & \\
\hline
\end{tabular}

Fonte: DCMS (2016, p. 22), com adaptações. [grifo nosso].

O código SIC 70.21 reconhece e apresenta o segundo lugar das relações públicas na indústria criativa: uma atividade econômica. Em outros termos, relações públicas é um setor produtivo, dotado de um conjunto de práticas específicas, as quais são realizadas para um 
determinado fim econômico. Vale dizer que, particularmente, nas relações públicas, a atividade precede a presença de um profissional. No dizer de Simões (1995), existe a atividade sem ter a necessidade de um profissional dotado de uma racionalidade técnica em relações públicas para exercê-la. Em que pese tal entendimento, o que se estabelece é a percepção de relações públicas como (i) profissão e (ii) atividade na indústria criativa. Mas é possível nomeadamente reconhecê-la como indústria criativa?

Ao retomar a metodologia do Reino Unido, a configuração da intensidade criativa é o método objetivo de perceber um setor como indústria criativa. A intensidade criativa é calculada pela proporção de ocupações criativas que estão em todos os setores da economia. Acima de $30 \%$, o setor é considerado indústria criativa.

Para o caso das relações públicas, enquanto atividade, isso se traduz da seguinte forma: qual o percentual de ocupações criativas (relações públicas profissão + demais ocupações criativas) está contido na atividade de relações públicas? O quadro abaixo apresenta as 10 atividades mais intensivas de criatividade no Reino Unido.

Quadro 04 - Intensidade criativa na indústria criativa no Reino Unido, primeiras posições, DCMS (2016).

\begin{tabular}{|l|l|l|}
\hline SIC & Descrição & $\begin{array}{l}\text { Intensidade criativa } \\
(\%)\end{array}$ \\
\hline 90.03 & Criação artística & 91.5 \\
\hline 74.30 & Atividades de tradução e interpretação & 82.2 \\
\hline 90.01 & Artes performáticas & 78.8 \\
\hline 74.20 & Atividades fotográficas & 77.8 \\
\hline 60.10 & Radiodifusão & 62.7 \\
\hline 74.10 & Atividades especializadas de design & 62.1 \\
\hline 71.11 & Atividades de arquitetura & 61.5 \\
\hline $\mathbf{7 0 . 2 1}$ & Relações Públicas e atividades de comunicação & $\mathbf{5 9 . 3}$ \\
\hline 58.14 & Publicação de revistas e outros e periódicos & 58.3 \\
\hline 90.02 & Atividades de apoio às artes performáticas & 56.8 \\
\hline
\end{tabular}

Fonte: DCMS (2016b, p. 08), com adaptações. [grifo nosso].

Nota-se que a intensidade criativa no SIC 70.21 "Relações Públicas e atividades de comunicação" é de 59,3\%, ou seja, de todas as ocupações que fazem parte dessa área, mais da metade são criativas, o que, para o modelo do DCMS, configura a atividade de relações públicas como uma indústria criativa. 
Portanto, o modelo do DCMS expõe o reconhecimento das relações públicas como indústria criativa através das (i) práticas profissionais, da (ii) ocupação como um trabalho técnico reconhecido, (iii) atividade econômica e (iv) pela intensidade criativa de tal atividade.

\section{ALGUNS RESULTADOS}

A indústria criativa destaca a criatividade como fator competitivo estratégico para bens e serviços estabelecidos em mercados dinâmicos que se utilizam desses fatores para criar elementos de diferenciação e agregação de valor. "Actividades no coração dessas trajetórias empresariais, como o design, a moda, o marketing, o 'branding' e a publicidade, contrastam com o núcleo-duro das atividades industriais e de serviços" (AUGUSTO MATEUS \& ASSOCIADOS, 2010, p. 16).

Essa indústria reconhece as competências e qualificações criativas como insumo básico, também destaca a configuração de processos diferenciados de concepção, criação, design e branding de bens e serviços como fundamentais na determinação do valor e, além disso, vê como central os profissionais dotados de capacidades diferenciadas para tais práticas (AUGUSTO MATEUS \& ASSOCIADOS, 2010).

Eis, então, que a discussão inicial das relações públicas entre a arte a ciência são rapidamente percebíveis nesses termos. As competências e qualificações criativas são notadas quando se utilizam de uma racionalidade científica, de determinadas técnicas e instrumentos para o desenvolvimento da atividade, mas não deixam de perceber e usar a capacidade criativa de quem as desenvolve.

Como é na maioria dos domínios de comunicação, o sucesso nas relações públicas não possui uma receita clara. É o resultado do uso de instrumentos adequados no momento certo. As várias campanhas de comunicação usadas por uma empresa ou uma organização obviamente têm a mensagem da empresa como um ponto de início, mas seu sucesso depende da capacidade dos especialistas de serem criativos (PALEA, 2010, p. 20, tradução nossa) ${ }^{12}$.

Por outro lado, a configuração de processos diferenciados de bens e serviços na determinação do valor também destaca que as relações públicas podem ir além de uma mera

\footnotetext{
${ }^{12}$ Tradução livre para: "As it is in most communication domains, success in PR does not have a clear recipe. It is the result of the use of suitable instruments at the right time. The various communication campaigns used by a company or an organization obviously have the company's message as a starting point, but their success depends on the specialists' capacity to be creative".
} 
promoção da organização/produto/marca. Relações públicas trazem, em sua prática, a combinação de elementos estratégicos, científicos e criativos. É por ordem desse processo com um rigor no método, mas aberto à criatividade, que as relações públicas são reconhecidas como "fornecedores de serviços criativos" (DAVIES; SIGTHORSSON, 2013). Esses prestadores de serviços dedicam o seu tempo e a sua propriedade intelectual para a criação de conteúdos criativos, gerando novas experiências significativas aos consumidores e agregando valor.

\begin{abstract}
Uma forma importante em que os bens simbólicos são transformados em experiências significativas para os consumidores é o branding. Uma marca é um bom exemplo de como a mistura de características tangíveis e intangíveis de produtos criativos ocorre na prática. É um bem simbólico que transmite significado e até carrega associações emocionais que não são inerentes às manifestações físicas da marca, como os produtos, o logo, a identidade visual da marca ou publicidades individuais (DAVIES; SIGTHORSSON, 2013, p. 133, tradução nossa) ${ }^{13}$.
\end{abstract}

Por fim, no que diz respeito aos profissionais dotados de capacidades diferenciadas, descritivamente se pode dizer que o relações públicas é como o especialista em iluminação de uma galeria de arte. "Sem a sua contribuição criativa para iluminar e tornar mais compreensível, podemos deixar de obter uma verdadeira compreensão ou esclarecimento sobre o mundo que nos rodeia" (GREEN, 2009, p. 146, tradução nossa) ${ }^{14}$. Já, normativamente, Palea (2010) diz que os especialistas de relações públicas devem trazer elementos de originalidade, procurar novas perspectivas e provar com cada campanha sua criatividade e profissionalismo. E do ponto de vista acadêmico-científico, como acentuou Kunsch (2006), compete aos investigadores das relações públicas apontar para novos paradigmas que sejam capazes de ler as mudanças (econômicas, tecnológicas, etc.), inscritas no campo da comunicação.

\title{
CONCLUSÃO
}

\footnotetext{
13 Tradução livre para: "One important way in which raw symbolic goods are turned into meaningful experiences for consumers is branding. A brand is a good example of how the mixture of tangible and intangible characteristics of creative products plays out in practice. It is a symbolic good that conveys meaning and even carries emotional associations that are not inherent in the physical manifestations of the brand, such as the products, the logo, the visual identity of the brand, or individual advertisements".

${ }^{14}$ Tradução livre para: "Without their creative contribution to illuminate and make more understandable, we can fail to gain true understanding of or enlightenment about the world around us".
} 
Após esse exercício reflexivo para perceber as relações públicas enquanto arte e ciência e posicioná-la na indústria criativa, podemos concluir que: A prática de relações públicas sempre terá regras, diretrizes, técnicas experimentadas, mas sempre será uma arte, uma habilidade, uma atividade que tem como insumo base a criatividade humana. $\mathrm{O}$ profissional de relações públicas é um artista que molda mensagens dentro de um contexto, tem compreensão dos públicos e do interesse potencial do mercado em uma história, pessoa ou marca (GREEN, 2009).

Assim, as relações públicas possuem a criatividade e a propriedade intelectual de quem a realiza como insumo (o conhecimento, as competências, a própria intuição). Encontram, nos seus processos, procedimentos que são científicos e metódicos (o diagnóstico, o planejamento, a pesquisa), mas, no entanto, entregam bens e serviços criados a partir da criatividade, da inventividade humana (as campanhas de relações públicas, o storytelling, os eventos, etc.). Relações públicas é, portanto, reconhecida, nesses termos, como creative service providers, algo como fornecedores de serviços criativos. Desta forma, conclui-se que não há exagero em dizer que o lugar das relações públicas, na indústria criativa, é como uma própria indústria criativa.

\section{REFERÊNCIAS}

AUGUSTO MATEUS \& ASSOCIADOS. O sector cultural e criativo em Portugal. Lisboa: Estudo para o Ministério da Cultura, 2010. (Relatório Final).

BENDASSOLLI, Pedro F. et al. Indústrias criativas: definição, limites e possibilidades. ERA, São Paulo, v. 49, n.1, jan./mar 2009, p. 10-18.

CONFERÊNCIA DAS NAÇÕES UNIDAS PARA O COMÉRCIO E DESENVOLVIMENTO UNCTAD. Relatório de Economia criativa - uma opção de desenvolvimento viável. UNCTAD: 2010. Disponível em: <http://unctad.org/pt/docs/ditctab20103_pt.pdf>. Acesso em: 04 de julho 2017.

DAVIES, Rosamund; SIGTHORSSON, Gauti. Introducing the creative industries. London: SAGE, 2013.

Department for Culture, Media and Sport - DCMS. Advertising. Mapping Documents, 2001. Disponível em: <https://www.gov.uk/government/publications/creative-industries-mappingdocuments-2001>. Acesso em: 10 de setembro 2017. 
Creative Industries Economic Estimates, January 2016. Disponível em:

<https://www.gov.uk/government/statistics/creative-industries-economic-estimates-january-2016>. Acesso em: 10 de setembro 2017.

. Creative Industries Economic Estimates Methodology, January 2016. Disponível em: <https://www.gov.uk/government/publications/creative-industries-economic-estimates-methodology>. Acesso em: 10 de setembro 2017.

GONÇALVES, Gisela. Ética das Relações Públicas. Coimbra: MinervaCoimbra, 2013.

GREEN, Andy. Creativity in public relations. London: Kogan Page, 2009.

GRUNIG, James; Todd, HUNT. Dirección de Relaciones Públicas. Barcelona: Gestión 2000, 2003.

HAZLETT, Kirk. Public Relations: Art or Science? Curry College, 2012. Disponível em: <https://shonaliburke.com/public-relations-art-or-science/>. Acesso em: 04 de julho 2017.

HOWKINS, John. Economia criativa: como ganhar dinheiro com ideias criativas. São Paulo: M. Books, 2013.

KUNSCH, Waldemar. Do mercado à academia: as relações públicas em seu primeiro centenário (1906-2006). Intercom - Revista Brasileira de Ciências da Comunicação, São Paulo, vol. 29, n. 2 , jul-dez, 2006, p. 55-87.

PALEA, Andina. Creativity in Public Relations. Professional communication and translation studies, Politehnica University of Timișoara, Romanian, v.3, 2010. Disponível em:

<http://www.cls.upt.ro/files/conferinte/proceedings/2010/04_Palea_2010.pdf >. Acesso em: 04 de julho 2017.

PROGRAMA DE PÓS-GRADUAÇÃO EM COMUNICAÇÃO E INDÚSTRIA CRIATIVA PPGCIC. Disponível em: <http://cursos.unipampa.edu.br/cursos/ppgcic/>. Acesso em: 04 de julho 2017.

SIMÕES, Roberto Porto. Relações Públicas: Função Política. São Paulo: Summus, 1995.

THROSBY, David. The concentric circles model of the cultural industries. Cultural Trends, vol. 17, n. 3, Sep. 2008, p. 147-164.

TURNEY, Michael. Is the practice of public relations art or science? Northern Kentucky University, 2009. Disponível em: <https://www.nku.edu/ turney/prclass/sections/art_science.html>. Acesso em: 04 de julho 2017.

VALIATI, Leandro; et al. Economia Criativa e da Cultura: conceitos, modelos teóricos e estratégias metodológicas. IN: VALIATI, Leandro; FIALHO, Ana Letícia do N. Porto Alegre: Editora da UFRGS/CEGOV, 2017. 
Bacharel em Comunicação Social, hab. Relações Públicas, pela Universidade Federal de Santa Maria (2002). Doutor em Desenvolvimento Regional pela Universidade de Santa Cruz do Sul (2014), com pesquisa no Exterior (bolsista PDSE/CAPES n ${ }^{\circ} 18017126$ ) na Universidade da Beira Interior UBI, Laboratório de Comunicação - LabCom, Covilhã, Portugal (2013). Pesquisador Ministério da Cultura/CNPq Edital No 80/2013, Economia Criativa no Brasil (2013-2015). Pesquisador CNPq Edital Chamada Universal 01/2016, Comunicação Pública e Indicadores Culturais (2017-2020). Professor do Programa de Pós-graduação em Comunicação e Indústria Criativa - mestrado profissional, Unipampa. Professor Adjunto do Curso de Relações Públicas - ênfase em produção cultural. Integrante do Grupo de Pesquisa "Processos e Práticas em Atividades Criativas e Culturais" (Unipampa/CNPq). Integrante do Observatório Missioneiro de Atividades Criativas e Culturais - OMiCult. Atualmente em afastamento para pós doutoramento na UI\&D - Centre for research in Communication, Information and Digital Culture CIC.DIGITAL Porto - Mestrado em Comunicação e Gestão das Indústrias

Criativas, Universidade do Porto, Portugal. Atua nas áreas de comunicação, cultura e desenvolvimento com ênfase em economia, política e indicadores culturais.

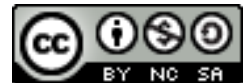

Esta obra está licenciado com uma Licença

Creative Commons Atribuição-NãoComercial-CompartilhaIgual 4.0 Internacional 